\title{
Tight sets and $m$-ovoids of generalised quadrangles
}

\author{
John Bamberg, Maska Law and Tim Penttila
}

\begin{abstract}
The concept of a tight set of points of a generalised quadrangle was introduced by S. E. Payne in 1987, and that of an $m$-ovoid of a generalised quadrangle was introduced by J. A. Thas in 1989, and we unify these two concepts by defining intriguing sets of points. We prove that every intriguing set of points in a generalised quadrangle is an $m$-ovoid or a tight set, and we state an intersection result concerning these objects. In the classical generalised quadrangles, we construct new $m$-ovoids and tight sets. In particular, we construct $m$-ovoids of $W(3, q), q$ odd, for all even $m$; we construct $(q+1) / 2$-ovoids of $W(3, q)$ for $q$ odd; and we give a lower bound on $m$ for $m$-ovoids of $H\left(4, q^{2}\right)$.
\end{abstract}

\section{Introduction}

This paper is concerned with certain sets of points and lines of finite generalised quadrangles which are extremal in some sense. A generalised quadrangle of order $(s, t)$ is an incidence structure of points and lines with the properties that every two points are incident with at most one line, every point is incident with $t+1$ lines, every line is incident with $s+1$ points, and for any point $P$ and line $\ell$ that are not incident, there is a unique point on $\ell$ collinear with $P$ (this is sometimes known as the "GQ axiom"). If $s=t$ we say the generalised quadrangle has order $s$. The standard reference is [14], however, we will not necessarily be using the same notation as that found there.

The examples of generalised quadrangles that play the most prominent role in this paper are the so-called classical generalised quadrangles $W(3, q), Q(4, q), H\left(3, q^{2}\right), Q^{-}(5, q)$ and $H\left(4, q^{2}\right)$. The first of these is the incidence structure of all totally isotropic points and totally isotropic lines with respect to a null polarity in $\mathrm{PG}(3, q)$, and is a generalised quadrangle of order $q$. The point/line dual of $W(3, q)$ is $Q(4, q)$, the parabolic quadric of $\mathrm{PG}(4, q)$, and is therefore a generalised quadrangle of order $q$ (see [14, 3.2.1]). The incidence structure of all points and lines of a non-singular Hermitian variety in $\mathrm{PG}\left(3, q^{2}\right)$, which forms the generalised quadrangle $H\left(3, q^{2}\right)$ of order $\left(q^{2}, q\right)$, has as its point/line dual the elliptic quadric $Q^{-}(5, q)$ in $\operatorname{PG}(5, q)$, which is a generalised quadrangle of order $\left(q, q^{2}\right)$ (see [14,3.2.3]). The remaining classical generalised quadrangle, $H\left(4, q^{2}\right)$, is the incidence structure of all points and lines of a nonsingular Hermitian variety in $\mathrm{PG}\left(4, q^{2}\right)$, and is of order $\left(q^{2}, q^{3}\right)$ (see $\left.[\mathbf{1 4}, 3.1 .1]\right)$.

The concept of a tight set of a generalised quadrangle was introduced by Payne in [15]. There, a set of points $\mathcal{T}$ of a generalised quadrangle of order $(s, t)$ is tight if that on average, each point $P$ of $\mathcal{T}$ is collinear with the maximum possible number of points of $\mathcal{T}$. If this maximum possible number is $s+i$, then we say the set $\mathcal{T}$ is $i$-tight. See Section 2 for an

2000 Mathematics Subject Classification. Primary 05B25, 51E12, 51E20, 51E23.

We thank the referees for their comprehensive and constructive advice. The first author was supported by an Australian Research Council Discovery-Projects Grant (no. DP0450695). The second author was supported by an Australian Research Council Discovery-Projects Grant (no. DP0209706). 
alternative definition of an $i$-tight set. It is not difficult to show that a 1-tight set is the set of points on a line, and Payne essentially determined the 2-tight sets in [15].

In Thas' paper [20], m-ovoids of generalised quadrangles were introduced as sets of points meeting every line in exactly $m$ points. The dual concept is an $m$-cover, that is, a set of lines such that every point lies on exactly $m$ lines of the set. These configurations generalise the older and more widely studied objects of ovoids and spreads (which are 1-ovoids and 1covers respectively). The complement of an ovoid in a generalised quadrangle of order $(s, t)$ is an $s$-ovoid, and apart from ovoids and complements of ovoids, there are few constructions of $m$-ovoids known.

A set of type $(a, b)$ of $\operatorname{PG}(d, q)$ is a set of points $\mathcal{P}$ such that each hyperplane intersects $\mathcal{P}$ in either $a$ or $b$ points. Such a point-set gives rise to a strongly regular graph and a two-weight code (see [5]). We observe in this paper, that in certain classical generalised quadrangles, $m$ ovoids and $i$-tight sets give rise to such sets of type $(a, b)$ (see Corollaries 4.6, 4.7, and 4.8). Hence the constructions of $m$-ovoids and $i$-tight sets of classical generalised quadrangles that we provide in this paper, give rise to strongly regular graphs and two-weight codes.

In the following two sections, we give brief accounts of the theory of tight sets and $m$-ovoids. In Section 4, we unify the notions of tight sets and m-ovoids in the context of intriguing sets of points, and prove some general combinatorial results of these objects. We show that every intriguing set of points in a generalised quadrangle is an $m$-ovoid or an $i$-tight set. We also show that in any generalised quadrangle, an $m$-ovoid intersects an $i$-tight set in $m i$ points, and we prove a partial converse of this theorem. In the classical generalised quadrangles, we construct new $m$-ovoids and new tight sets. In Section 5 , we construct an infinite family of $m$-ovoids of $W(3, q), q$ odd, for all even $m$. We then follow this construction in Section 6 with a construction of $(q+1) / 2$-ovoids of $W(3, q)$ for $q$ odd. By duality, these $m$-ovoids are hemisystems (of lines) of $Q(4, q)$. In Section 7 , we show that $H\left(4, q^{2}\right)$ does not have $m$-ovoids for $m \leq \sqrt{q}$. Finally, we provide some other examples of intriguing sets and conclude with some open problems.

\section{Tight Sets}

The concept of a tight set can be made more explicit with the following definition. A set of points $\mathcal{T}$ of a generalised quadrangle of order $(s, t)$ is an $i$-tight set if for every point $P$ in $\mathcal{T}$, there are $s+i$ points of $\mathcal{T}$ collinear with $P$, and for every point $P$ not in $\mathcal{T}$, there are $i$ points of $\mathcal{T}$ collinear with $P$. We say that a set of points is tight if it is $i$-tight for some $i$. By [15, II.1], the size of an $i$-tight set of a generalised quadrangle of order $(s, t)$ is $i(s+1)$. Tight sets exhibit the following nice properties, which we recast and summarise from Payne's original paper.

Lemma 2.1 (S. E. Payne 1987, [15, II.2.]). Let $\mathcal{S}$ be a generalised quadrangle of order $(s, t)$. Let $\mathcal{A}$ and $\mathcal{B}$ be $i$-tight and $j$-tight sets of points of $\mathcal{S}$, respectively. Then:

(a) if $\mathcal{A} \subseteq \mathcal{B}$, then $\mathcal{B} \backslash \mathcal{A}$ is $(j-i)$-tight;

(b) if $\mathcal{A}$ and $\mathcal{B}$ are disjoint, then $\mathcal{A} \cup \mathcal{B}$ is $(i+j)$-tight;

(c) the set of points of $\mathcal{S}$ form an $($ st +1$)$-tight set, and the empty set is 0-tight.

It is worth noting that $Q(4, q)$ is a $\left(q^{2}+1\right)$-tight set of $Q^{-}(5, q)$ and that $H\left(3, q^{2}\right)$ is a $\left(q^{3}+1\right)$-tight set of $H\left(4, q^{2}\right)$.

\section{3. $m$-ovoids}

As for tight sets, we can consider $m$-ovoids in terms of the number of points of the set collinear with any given point of the generalised quadrangle. Thus an $m$-ovoid of a generalised quadrangle of order $(s, t)$ is a set of points $\mathcal{O}$ such that for every point $P$ in $\mathcal{O}$, there are 
$(t+1)(m-1)+1$ points of $\mathcal{O}$ collinear with $P$, and for every point $P$ not in $\mathcal{O}$, there are $(t+1) m$ points of $\mathcal{O}$ collinear with $P$. By [20, Lemma 1], the size of an $m$-ovoid of a generalised quadrangle of order $(s, t)$ is $m(s t+1)$. The following properties, analogous to those given in Lemma 2.1 for tight sets, are straightforward consequences of the definition of an $m$-ovoid.

LEMMA 3.1. Let $\mathcal{S}$ be a generalised quadrangle of order $(s, t)$. Let $\mathcal{A}$ and $\mathcal{B}$ be an $m$-ovoid and $n$-ovoid of $\mathcal{S}$ respectively. Then:

(a) if $\mathcal{A} \subseteq \mathcal{B}$, then $\mathcal{B} \backslash \mathcal{A}$ is an $(n-m)$-ovoid;

(b) if $\mathcal{A}$ and $\mathcal{B}$ are disjoint, then $\mathcal{A} \cup \mathcal{B}$ is an $(n+m)$-ovoid;

(c) the set of points of $\mathcal{S}$ form an $(s+1)$-ovoid, and the empty set is a 0-ovoid.

As mentioned in the introduction, apart from ovoids and complements of ovoids, there are few constructions of $m$-ovoids known. For $q$ even, K. Drudge [8] constructed a 2-cover of $W(3, q)$, and so a 2-ovoid of $Q(4, q)$. Recently, A. Cossidente and the third author [7] constructed an $m$-ovoid of $H(3,25)$ for $m \in\{5,6,20,21\}$ and a $(q+1) / 2$-ovoid of $Q^{-}(5, q)$ for all odd $q$. By intersection, the latter gives a $(q+1) / 2$-ovoid of $Q(4, q), q$ odd. It is well-known that there are no 1 -ovoids of $W(3, q)$ for $q$ odd (see $[\mathbf{1 4}]$ or $[\mathbf{1 9}]$ ).

\section{Combinatorics of intriguing sets}

A subset $\mathcal{I}$ of points of a generalised quadrangle is intriguing if over all points $P$ in $\mathcal{I}$, the number of points in $\mathcal{I}$ collinear with $P$ is constant, and over all points $P$ not in $\mathcal{I}$, the number of points in $\mathcal{I}$ collinear with $P$ is also constant. Necessarily, these two constants must be different. Throughout this paper, we will use the notation $\mathcal{B}^{\perp}$ to denote the set of points collinear with each point in $\mathcal{B}$ (we write $P^{\perp}$ for $\{P\}^{\perp}$ ). Hence $\mathcal{I}$ is intriguing if

$$
\left|P^{\perp} \cap \mathcal{I}\right|= \begin{cases}h_{1} & \text { if } P \in \mathcal{I}, \\ h_{2} & \text { otherwise }\end{cases}
$$

for some constants $h_{1}$ and $h_{2}$. Now we saw in the preceding sections that $m$-ovoids and $i$-tight sets are intriguing. The converse is also true.

THEOREM 4.1. An intriguing set of a generalised quadrangle is an m-ovoid or is an i-tight set.

Proof. Let $\mathcal{I}$ be an intriguing set of a generalised quadrangle of order $(s, t)$, let $\ell$ be a line and let $m$ be the number of points on $\ell$ that are in $\mathcal{I}$. Since $\mathcal{I}$ is intriguing, there exist constants $h_{1}$ and $h_{2}$ such that $\left|P^{\perp} \cap \mathcal{I}\right|=h_{1}$ for all $P \in \mathcal{I}$, and $\left|P^{\perp} \cap \mathcal{I}\right|=h_{2}$ for all $P \notin \mathcal{I}$. There are $s+1-m$ points on $\ell$ that are not in $\mathcal{I}$, and for each of these points $P$, there are $h_{2}-m$ points of $\mathcal{I}$ not on $\ell$ that are collinear with $P$. So there are $(s+1-m)\left(h_{2}-m\right)$ points of $\mathcal{I}$ not on $\ell$ collinear with points of $\ell \backslash(\ell \cap \mathcal{I})$ (by the "GQ axiom"). On the other hand, there are $m$ points on $\ell$ that are in $\mathcal{I}$, and so there are $m\left(h_{1}-m\right)$ points of $\mathcal{I}$ not on $\ell$ that are collinear with points of $\ell \cap \mathcal{I}$. So $|\mathcal{I}|-m$ is equal to $(s+1-m)\left(h_{2}-m\right)+m\left(h_{1}-m\right)$ and hence

$$
|\mathcal{I}|-(s+1) h_{2}=m\left(h_{1}-h_{2}-s\right) .
$$

If $h_{1}-h_{2}=s$, then $|\mathcal{I}|=(s+1) h_{2}$ and $\mathcal{I}$ is $h_{2}$-tight. If $h_{1}-h_{2} \neq s$, then

$$
m=|\ell \cap \mathcal{I}|=\frac{|\mathcal{I}|-(s+1) h_{2}}{h_{1}-h_{2}-s}
$$

for all lines $\ell$, and so $\mathcal{I}$ is an $m$-ovoid. 
COROLLARY 4.2. Let $\mathcal{I}$ be an intriguing set of points of a generalised quadrangle $\mathcal{S}$. Let $h_{1}$ be the number of points of $\mathcal{I}$ collinear with a point of $\mathcal{I}$, and let $h_{2}$ be the number of points of $\mathcal{I}$ collinear with a point not in $\mathcal{I}$. Then $\mathcal{I}$ is an $m$-ovoid if $h_{1}<h_{2}$, and is a tight set otherwise.

THEOREM 4.3. Let $\mathcal{S}$ be a generalised quadrangle, let $\mathcal{O}$ be an $m$-ovoid of $\mathcal{S}$ and let $\mathcal{T}$ be an $i$-tight set of $\mathcal{S}$. Then $\mathcal{O}$ and $\mathcal{T}$ intersect in mi points.

Proof. Let $\mathcal{P}$ be the point-set of a generalised quadrangle $\mathcal{S}$ of order $(s, t)$, and let $A$ be the adjacency matrix of the collinearity graph of $\mathcal{S}$ (with the loops removed). Consider $A$ as a linear map on the vector space of functions $\mathbb{R}^{\mathcal{P}}$; i.e., $A$ maps a function $\varphi$ to the function $P \mapsto \sum\{\varphi(Q): Q \sim P, \quad Q \neq P\}$. For all subsets $\mathcal{B}$ of $\mathcal{P}$, let $\chi_{\mathcal{B}}$ denote the characteristic function on $\mathcal{B}$ :

$$
\chi_{\mathcal{B}}(P)= \begin{cases}1 & \text { if } P \in \mathcal{B} \\ 0 & \text { otherwise. }\end{cases}
$$

Note that it is the inner product $\chi_{\mathcal{O}} \cdot \chi_{\mathcal{T}}$ that we wish to calculate, as this is precisely the size of the intersection of $\mathcal{O}$ and $\mathcal{T}$. Now by the definition of an $m$-ovoid, we have

$$
A \chi_{\mathcal{O}}=-(t+1) \chi_{\mathcal{O}}+(t+1) m \eta
$$

where $\eta$ is the constant function with value 1 . Similarly, by the definition of an $i$-tight set, we have

$$
A \chi_{\mathcal{T}}=(s-1) \chi_{\mathcal{T}}+i \eta
$$

Since the valency of each point of the collinearity graph of $\mathcal{S}$ is $s(t+1)$, we have

$$
A \eta=s(t+1) \eta
$$

Now

$$
A\left(\chi_{\mathcal{O}}-\frac{m}{s+1} \eta\right)=-(t+1) \chi_{\mathcal{O}}+(t+1) m \eta-\frac{m s(t+1)}{s+1} \eta=-(t+1)\left(\chi_{\mathcal{O}}-\frac{m}{s+1} \eta\right)
$$

and

$$
A\left(\chi_{\mathcal{T}}-\frac{i}{s t+1} \eta\right)=(s-1) \chi_{\mathcal{T}}+i \eta-\frac{i s(t+1)}{s t+1} \eta=(s-1)\left(\chi_{\mathcal{T}}-\frac{i}{s t+1} \eta\right) .
$$

Therefore $\chi_{\mathcal{O}}-\frac{m}{s+1} \eta$ and $\chi_{\mathcal{T}}-\frac{i}{s t+1} \eta$ are eigenvectors of $A$, with different eigenvalues, and hence they must be orthogonal. So

$$
\left(\chi_{\mathcal{O}}-\frac{m}{s+1} \eta\right) \cdot\left(\chi_{\mathcal{T}}-\frac{i}{s t+1} \eta\right)=0
$$

By rearranging we see that

$$
\chi_{\mathcal{O}} \cdot \chi_{\mathcal{T}}=m i\left(\frac{\eta \cdot \chi_{\mathcal{T}}}{(s+1) i}-\frac{\eta \cdot \eta}{(s+1)(s t+1)}+\frac{\chi_{\mathcal{O}} \cdot \eta}{(s t+1) m}\right) .
$$

We can count in the generalised quadrangle $\mathcal{S}$ and obtain

$$
\begin{aligned}
\eta \cdot \chi_{\mathcal{T}} & =|\mathcal{T}|=(s+1) i \\
\chi_{\mathcal{O}} \cdot \eta & =|\mathcal{O}|=(s t+1) m \\
\eta \cdot \eta & =|\mathcal{P}|=(s+1)(s t+1) .
\end{aligned}
$$

So $|\mathcal{O} \cap \mathcal{T}|=\chi_{\mathcal{O}} \cdot \chi_{\mathcal{T}}=m i$ and hence $\mathcal{O}$ and $\mathcal{T}$ intersect in $m i$ points. 
We now state some interesting corollaries of this result, some of which offer different proofs of old results. The first of these concerns regular pairs of points of a generalised quadrangle. In a generalised quadrangle of order $(s, t)$, a hyperbolic line is a set of points of the form $\{P, Q\}^{\perp \perp}$ for some pair of non-collinear points $P$ and $Q$. Every hyperbolic line has at most $t+1$ points (by the "GQ axiom"), and if equality occurs, then we say that the pair $(P, Q)$ defining the hyperbolic line is a regular pair (see [14, pp. 4]). By $[\mathbf{1 4}, 1.6 .1]$, every pair of non-collinear points of $W(3, q)$ is a regular pair.

Corollary 4.4 (S. E. Payne and J. A. Thas 1984, $[\mathbf{1 4}, 1.8 .4]$ ). Let $\mathcal{S}$ be a classical generalised quadrangle of order $s$, with $s$ even, let $\mathcal{O}$ be an ovoid of $\mathcal{S}$, and suppose there is a regular pair of non-collinear points $(P, Q)$. Then $\left|\mathcal{O} \cap\{P, Q\}^{\perp \perp}\right|,\left|\mathcal{O} \cap\{P, Q\}^{\perp}\right| \in\{0,2\}$ and $\left|\mathcal{O} \cap\left(\{P, Q\}^{\perp} \cup\{P, Q\}^{\perp \perp}\right)\right|=2$.

Proof. This follows from the fact due to Payne in [15] that $\{P, Q\}^{\perp} \cup\{P, Q\}^{\perp \perp}$ is 2-tight (the division $\left|\mathcal{O} \cap\{P, Q\}^{\perp \perp}\right|=\left|\mathcal{O} \cap\{P, Q\}^{\perp}\right|=1$ being impossible since every point of $\{P, Q\}^{\perp}$ is collinear with every point of $\{P, Q\}^{\perp \perp}$, and so some line of $\mathcal{S}$ then contains 2 points of $\mathcal{O}$ ).

Corollary 4.5 (J. A. Thas 1972, [18]). If $\mathcal{O}$ is an ovoid of $W(3, q)$, where $q$ is even, then $\mathcal{O}$ is an ovoid of $\mathrm{PG}(3, q)$.

Proof. First note that every line of $\operatorname{PG}(3, q)$ is either a line of $W(3, q)$, and hence meets $\mathcal{O}$ in a single point, or is a hyperbolic line of $W(3, q)$. By Corollary 4.4 , every hyperbolic line meets $\mathcal{O}$ in 0 or 2 points. Therefore, $\mathcal{O}$ is a cap of $\mathrm{PG}(3, q)$, and hence an ovoid, as it has size $q^{2}+1$.

Recall from the introduction the notion of a set of type $(a, b)$ with respect to hyperplanes and their connections to strongly regular graphs and two-weight codes. Note that an $m$-ovoid of $W(3, q)$ is a set of $m\left(q^{2}+1\right)$ points of $\mathrm{PG}(3, q)$ of type $(m(q+1)-q, m(q+1))$ with respect to planes. We now observe similar results for other classical generalised quadrangles.

Corollary 4.6. An m-ovoid $\mathcal{O}$ of $Q^{-}(5, q)$ gives rise to a set of points of $\mathrm{PG}(5, q)$ of type $\left(m\left(q^{2}+1\right)-q^{2}, m\left(q^{2}+1\right)\right)$ with respect to hyperplanes.

Proof. For a point $P$ of $Q^{-}(5, q),\left|P^{\perp} \cap \mathcal{O}\right|$ is $m\left(q^{2}+1\right)-q^{2}$ if $P \in \mathcal{O}$ and $m\left(q^{2}+1\right)$ if $P \notin \mathcal{O}$. For a point $P$ of $\operatorname{PG}(5, q)$ not of $Q^{-}(5, q), P^{\perp} \cap Q^{-}(5, q)$ is a subquadrangle $Q(4, q)$, and so is $\left(q^{2}+1\right)$-tight. Hence $\left|P^{\perp} \cap \mathcal{O}\right|=m\left(q^{2}+1\right)$.

Corollary 4.7. An m-ovoid $\mathcal{O}$ of $H\left(4, q^{2}\right)$ gives rise to a set of points of $\mathrm{PG}\left(4, q^{2}\right)$ of type $\left(m\left(q^{3}+1\right)-q^{3}, m\left(q^{3}+1\right)\right)$ with respect to hyperplanes.

Proof. For a point $P$ of $H\left(4, q^{2}\right),\left|P^{\perp} \cap \mathcal{O}\right|$ is $m\left(q^{3}+1\right)-q^{3}$ if $P \in \mathcal{O}$ and $m\left(q^{3}+1\right)$ if $P \notin \mathcal{O}$. For a point $P$ of $\mathrm{PG}\left(4, q^{2}\right)$ not of $H\left(4, q^{2}\right), P^{\perp} \cap H\left(4, q^{2}\right)$ is a subquadrangle $H\left(3, q^{2}\right)$, and so is $\left(q^{3}+1\right)$-tight. Hence $\left|P^{\perp} \cap \mathcal{O}\right|=m\left(q^{3}+1\right)$.

COROllary 4.8. An $i$-tight set $\mathcal{T}$ of $H\left(3, q^{2}\right)$ gives rise to a set of points of $\mathrm{PG}\left(3, q^{2}\right)$ of type $\left(i, i+q^{2}\right)$ with respect to planes.

Proof. For a point $P$ of $H\left(3, q^{2}\right),\left|P^{\perp} \cap \mathcal{T}\right|$ is $i+q^{2}$ if $P \in \mathcal{T}$ and $i$ if $P \notin \mathcal{T}$. For a point $P$ of $\mathrm{PG}\left(3, q^{2}\right)$ not of $H\left(3, q^{2}\right), P^{\perp} \cap H\left(3, q^{2}\right)$ is an ovoid. Hence $\left|P^{\perp} \cap \mathcal{T}\right|=i$.

Corollary 4.9. Let $\mathcal{O}$ be a $(q+1) / 2$-ovoid of $Q^{-}(5, q)$. Let $\pi$ be a plane of $\mathrm{PG}(5, q)$ meeting $Q^{-}(5, q)$ in a non-degenerate conic $\mathcal{C}$. Let $\mathcal{T}=\mathcal{C} \cup\left(\pi^{\perp} \cap Q^{-}(5, q)\right)$. Then $|\mathcal{T} \cap \mathcal{O}|=q+1$.

Proof. By Payne [15, II.4(ii)], $\mathcal{T}$ is a 2-tight set.

In the following Theorem, we use the fact that the collinearity graph of a generalised quadrangle of order $(s, t)$ is a strongly regular graph and hence the adjacency matrix has exactly three eigenvalues. 
THEOREM 4.10. Let $\mathcal{S}$ be a classical generalised quadrangle. If there is an $m$-ovoid of $\mathcal{S}$ and $\mathcal{B}$ is a set of $(s+1) i$ points of $\mathcal{S}$ such that $|\mathcal{B} \cap \mathcal{O}|=$ mi for all $m$-ovoids $\mathcal{O}$ of $\mathcal{S}$, then $\mathcal{B}$ is $i$-tight. If there is an $i$-tight set of $\mathcal{S}$ and $\mathcal{B}^{\prime}$ is a set of $m(s t+1)$ points of $\mathcal{S}$ such that $\left|\mathcal{B}^{\prime} \cap \mathcal{T}\right|=$ mi for all $i$-tight sets $\mathcal{T}$ of $\mathcal{S}$, then $\mathcal{B}^{\prime}$ is an m-ovoid.

Proof. Since the automorphism group $G$ of $\mathcal{S}$ has permutation rank three on the points of $\mathcal{S}$, the three eigenspaces corresponding to the adjacency matrix of the collinearity graph of $\mathcal{S}$ are irreducible. The eigenspace $W$ corresponding to the eigenvalue $-(t+1)$ is the orthogonal complement of the sum of the other two eigenspaces. Similarly, the eigenspace $U$ corresponding to the eigenvalue $s-1$ is the orthogonal complement of the sum of the other two eigenspaces.

It follows from the details of the proof of Theorem 4.3 that a set $\mathcal{O}$ of points is an $m$-ovoid if and only if $\chi_{\mathcal{O}}-\frac{m}{s+1} \eta$ is an eigenvector of the adjacency matrix with eigenvalue $-(t+1)$, and that a set $\mathcal{T}$ of points is $i$-tight if and only if $\chi_{\mathcal{T}}-\frac{i}{s t+1} \eta$ is an eigenvector of the adjacency matrix with eigenvalue $s-1$. Suppose there exists an $m$-ovoid of $\mathcal{S}$ and $\mathcal{B}$ is a set of $(s+1) i$ points such that $|\mathcal{B} \cap \mathcal{O}|=m i$ for all $m$-ovoids $\mathcal{O}$ of $\mathcal{S}$. To show that $\mathcal{B}$ is $i$-tight, it is sufficient to show that $\chi_{\mathcal{B}}-\frac{i}{s t+1} \eta$ is orthogonal to $W$, since by hypothesis, $|\mathcal{B}|=(s+1) i$ and hence $\chi_{\mathcal{B}}-\frac{i}{s t+1} \eta$ is orthogonal to the eigenspace corresponding to $\eta$. Since $W$ is irreducible, this condition is equivalent to $\chi_{\mathcal{B}}-\frac{i}{s t+1} \eta$ being orthogonal to $\chi_{\mathcal{O}}-\frac{m}{s+1} \eta$ over all $m$-ovoids $\mathcal{O}$; which follows from our assumption.

The proof of the second part of this result is similar.

A Cameron-Liebler line class of $\mathrm{PG}(3, q)$ with parameter $x$ is a set $\mathcal{L}$ of lines of $\mathrm{PG}(3, q)$ that shares precisely $x$ lines with each spread of $\mathrm{PG}(3, q)$ (see $[\mathbf{4}]$ ).

Corollary 4.11. A Cameron-Liebler line class $\mathcal{L}$ in $\mathrm{PG}(3, q)$ with parameter $x$ gives an $x$-tight set of $Q(4, q)$.

Proof. First note that every spread of $W(3, q)$ is a spread of $\mathrm{PG}(3, q)$. The set of lines of $\mathcal{L}$ in $W(3, q)$ meets each spread of $W(3, q)$ in $x$ lines and so is an $x$-tight set of $Q(4, q)$.

So by the above corollary, there arise $\left(q^{2}+1\right) / 2$-tight sets of $Q(4, q)$ for $q$ odd, by the construction of $[4]$.

\section{5. $m$-ovoids in $W(3, q), m$ even and $q$ odd}

In this section we will use ovoids of $\mathrm{PG}(3, q)$ to construct new $m$-ovoids of $W(3, q)$, when $q$ is odd. This is despite the fact that there are no ovoids of $W(3, q), q$ odd (see [18]). It is well known that every ovoid of $\mathrm{PG}(3, q), q$ odd, is an elliptic quadric (see Barlotti [2] and Panella [13]), although we require only the synthetic properties of such ovoids for our construction. We now give a construction of an infinite family of 2-ovoids of $W(3, q)$, for $q$ odd.

THEOREM 5.1. Let $q$ be odd, let $\mathcal{O}$ be an ovoid of $\mathrm{PG}(3, q)$, let $\rho$ be the associated polarity of $\mathcal{O}$, and let $\sigma$ be a null polarity of $\mathrm{PG}(3, q)$ such that at each point $P$ of $\mathcal{O}$, there is precisely one tangent line at $P$ which is self-polar with respect to $\sigma$. Then $\mathcal{O}^{\rho \sigma}$ is an ovoid of $\operatorname{PG}(3, q)$ disjoint from $\mathcal{O}$, and $\mathcal{O} \cup \mathcal{O}^{\rho \sigma}$ is a 2-ovoid of $W(3, q)$.

Proof. Since the product of $\rho$ and $\sigma$ is a collineation, it follows that $\mathcal{O}^{\rho \sigma}$ is an ovoid of $\operatorname{PG}(3, q)$. If $X$ is a point of $\mathcal{O}$, and $\ell$ is the unique tangent line at $X$ which is self-polar with respect to $\sigma$, then $\ell$ lies in $X^{\rho}$ and so $X^{\rho \sigma} \in \ell^{\sigma}=\ell$. Since $\ell$ is the unique self-polar tangent line on $X$ in $X^{\rho}$, we have that $X$ is not the carrier of the pencil of self-polar (with respect to $\sigma)$ lines containing $\ell$ in $X^{\rho}$. This carrier point is equal to $X^{\rho \sigma}$ and hence $X \neq X^{\rho \sigma}$. Since $\ell$ is tangent to $\mathcal{O}$, we have that $X^{\rho \sigma} \notin \mathcal{O}$, and therefore our two ovoids $\mathcal{O}$ and $\mathcal{O}^{\rho \sigma}$ are disjoint.

Suppose $\ell$ is a line of $W(3, q)$. We will show that $\ell$ meets $\mathcal{O} \cup \mathcal{O}^{\rho \sigma}$ in precisely two points. We have three cases: 
$\ell$ is exterior to $\mathcal{O}$ : Then there are two distinct planes $\pi_{1}$ and $\pi_{2}$ of $\mathrm{PG}(3, q)$ which intersect in $\ell$ and which are both tangent to $\mathcal{O}$. Since $\mathcal{O}^{\rho \sigma}$ is an ovoid, we know that $\ell$ meets $\mathcal{O}^{\rho \sigma}$ in at most two points. Now $\pi_{1}^{\sigma}$ and $\pi_{2}^{\sigma}$ are two points on $\ell$ which lie on $\mathcal{O}^{\rho \sigma}$, and so $\ell$ meets $\mathcal{O}^{\rho \sigma}$ in precisely two points.

$\ell$ is tangent to $\mathcal{O}$ : By the construction of the ovoid $\mathcal{O}^{\rho \sigma}$, we have that $\ell$ meets it in precisely one point, namely $\left((\ell \cap \mathcal{O})^{\rho}\right)^{\sigma}$. Therefore $\ell$ meets $\mathcal{O} \cup \mathcal{O}^{\rho \sigma}$ in precisely two points.

$\ell$ is secant to $\mathcal{O}$ : We show that $\ell$ is exterior to $\mathcal{O}^{\rho \sigma}$. If we suppose the contrary, then there is a point $X$ of $\mathcal{O}$ such that $\ell$ is incident with $X^{\rho \sigma}$. By applying the polarity $\sigma$, we find that $\ell$ is contained in $X^{\rho}$, which is a contradiction as $X^{\rho}$ is the tangent plane to $X$ and $\ell$ is secant to $\mathcal{O}$. Therefore $\ell$ meets $\mathcal{O} \cup \mathcal{O}^{\rho \sigma}$ in precisely two points.

Such a null polarity $\sigma$ satisfying the hypotheses of the above theorem exists, as we explain below. Given an elliptic orthogonal polarity $\rho$ and a null polarity $\sigma$ of $\operatorname{PG}(3, q)$, with $q$ odd, either every tangent pencil of lines with respect to the underlying elliptic quadric $\mathcal{O}$ of $\rho$ contains a unique absolute line with respect to $\sigma$ or exactly two tangent pencils of lines with respect to $\mathcal{O}$ have every member absolute with respect to $\sigma$. In each case, there is a unique conjugacy class of such polarities $\sigma$ under the centraliser of $\rho$ in $\operatorname{P\Gamma L}(4, q)$.

By [17], the image of the tangent lines to $\mathcal{O}$ under the Klein correspondence is the unique 1-system of the Klein quadric, which can also be constructed by applying field reduction to a conic $\mathcal{C}$ in $\operatorname{PG}\left(2, q^{2}\right)$. Every hyperplane of $\mathrm{PG}(5, q)$ contains a unique line $\ell$ of $\mathrm{PG}\left(2, q^{2}\right)$ (after field reduction); if $\ell$ is tangent to $\mathcal{C}$ then the hyperplane is degenerate; if $\ell$ is secant to $\mathcal{C}$, then its image after field reduction is a hyperbolic 3-space, which lies on two degenerate hyperplanes and $q-1$ non-degenerate hyperplanes; if $\ell$ is external to $\mathcal{C}$, then its image after field reduction is an elliptic 3 -space, which lies on $q+1$ non-degenerate hyperplanes. Moreover, by Witt's theorem, the stabiliser of $\mathcal{C}$ acts transitively on both the secant lines and the external lines; and it follows easily that the stabiliser of the 1-system acts transitively on non-degenerate hyperplanes containing the image after field reduction of a secant line and on non-degenerate hyperplanes containing the image after field reduction of an external line. Applying the Klein correspondence again shows that our claim above that there exists a null polarity $\sigma$ such that at each point $P$ of $\mathcal{O}$, there is precisely one tangent line at $P$ which is self-polar with respect to $\sigma$.

Corollary 5.2. For $q$ odd, there is a partition of $W(3, q)$ into 2-ovoids and hence for all even $m$, there exist $m$-ovoids of $W(3, q)$ (for some $q$ ).

Proof. Let $f: \operatorname{GF}\left(q^{4}\right) \times \operatorname{GF}\left(q^{4}\right) \rightarrow \operatorname{GF}\left(q^{4}\right)$ be the Hermitian form $f(x, y)=x y^{q^{2}}$, let $T: \operatorname{GF}\left(q^{4}\right) \rightarrow \operatorname{GF}(q)$ be the (relative) trace, so that $T(x)=x+x^{q}+x^{q^{2}}+x^{q^{3}}$. Let $\gamma \in \operatorname{GF}\left(q^{4}\right)$. Then $T(\gamma f)$ is a symmetric bilinear form of minus type if $\gamma^{q^{2}}=\gamma$ and is an alternating form if $\gamma^{q^{2}}=-\gamma$. Consider the group $C$ given by $\left\{x \mapsto \lambda x \mid \lambda^{2\left(q^{2}+1\right)}=1\right\}$ (note that the elements of $C$ are isometries with respect to the aforementioned symmetric bilinear and alternating forms). Then $C$ induces a cyclic subgroup $G$ of $\operatorname{PGL}(4, q)$ of order $q^{2}+1$, which by Ebert [11], has $q+1$ orbits on $\mathrm{PG}(3, q)$ which are all elliptic quadrics. Each orbit $\mathcal{O}$ corresponds to a choice of $\gamma$ with $\gamma^{q^{2}}=\gamma$ (namely $\mathcal{O}$ is the set of singular points of $T(\gamma f)$ ). Let $\rho=\rho_{\gamma}$ be the corresponding polarity. Fix $\gamma \in \mathrm{GF}\left(q^{4}\right)$ with $\gamma^{q^{2}}=-\gamma$ and let $\sigma$ be the corresponding (null) polarity. Then $G$ commutes with both $\rho$ and $\sigma$ and so with $\rho \sigma$ (since the elements of $G$ are projective isometries with respect to $\rho$ and $\sigma$ ). Thus $\rho \sigma$ permutes the orbits of $G$. Hence, if we apply the construction of Theorem 5.1 to an orbit $\mathcal{O}$ of $G$, then $\mathcal{O}^{\rho \sigma}$ is also an orbit of $G$. In this way, the orbit partition of $G$ induces a partition of $W(3, q)$ into 2-ovoids. 


\section{Hemisystems of lines of $Q(4, q)$}

In this section we give a construction of an infinite family of $(q+1) / 2$-covers of $Q(4, q)$. A $(q+1) / 2$-cover of the generalised quadrangle $H\left(3, q^{2}\right)$ was termed a hemisystem by Segre in [16]. Since an $m$-cover of a generalised quadrangle gives rise to an $m$-ovoid of the point/line dual generalised quadrangle, we will use the term hemisystem for both a $(q+1) / 2$-cover and for a $(q+1) / 2$-ovoid, stating "of lines" or "of points" when the context is not clear. Here we construct hemisystems of lines of $Q(4, q)$, giving rise to hemisystems of points of $W(3, q)$.

The construction involves sets of lines of $\mathrm{PG}(3, q)$ called reguli. That is, let $\mathcal{R}$ be a nonempty set of mutually skew lines of $\operatorname{PG}(3, q)$ such that through each point of each line of $\mathcal{R}$ there is a line meeting each line of $\mathcal{R}$ in exactly one point (such a line is called a transversal of $\mathcal{R}$ ), and such that through each point of each such transversal of $\mathcal{R}$ there is a line of $\mathcal{R}$. Then $\mathcal{R}$ is called a regulus. The set of all transversals of the regulus $\mathcal{R}$ forms a regulus, called the opposite regulus, and any regulus necessarily consists of $q+1$ lines.

THEOREM 6.1. Let $\Pi$ be a partition of the lines of $Q(4, q)$ into reguli, which is closed under the taking of opposite reguli. Then $q$ is odd and $2^{\left(q^{2}+1\right) / 2}$ hemisystems of lines of $Q(4, q)$ (and so the same number of hemisystems of points of $W(3, q)$ ) arise, by taking one regulus from each of the regulus-opposite regulus pairs in $\Pi$, and forming the union of these chosen reguli.

Proof. Consider the set of all lines through some point $P$ of $Q(4, q)$. Choosing one regulus from each regulus-opposite regulus pair in $\Pi$ clearly amounts to selecting exactly half of the $q+1$ lines through $P$, since for every line $\ell$ through $P$ there exists a second line $\ell^{\prime}$ through $P$ which lies in the opposite regulus to the unique regulus in $\Pi$ containing $\ell$. The number of reguli in $\Pi$ is $q^{2}+1$, and so the number of hemisystems of lines arising follows from the choice of one regulus from each of the $\left(q^{2}+1\right) / 2$ regulus-opposite regulus pairs in $\Pi$.

Below we give examples for which we get partitions $\Pi$ of the lines of $Q(4, q)$ into reguli, with the desired properties stated in the theorem above. The critical observation to note is that the points of a non-degenerate hyperbolic quadric $\mathcal{Q}^{+}$in $\operatorname{PG}(3, q)$ are the points on some regulus $\mathcal{R}$. The lines of $\mathcal{Q}^{+}$are then the lines of $\mathcal{R}$ together with the lines of the opposite regulus of $\mathcal{R}$.

Example 1. Let $\ell$ be a line exterior to the parabolic quadric $\mathcal{Q}$ in $\mathrm{PG}(4, q), q$ odd. Let $\pi$ be the polar plane to $\ell$. Then $\pi \cap \mathcal{Q}=\mathcal{C}$ is a conic of $\pi$. Consider the set of hyperbolic quadrics containing $\mathcal{C}$ together with the hyperbolic quadrics spanning a space containing $\ell$. Then the set of reguli contained in the hyperbolic quadrics in this set forms a partition $\Pi$ satisfying the hypotheses of Theorem 6.1. To see this, let $m$ be a line on $\mathcal{Q}$. Then we have two cases; either $m$ meets $\pi$ or is skew to $\pi$. In the former case, $m$ lies in a hyperbolic quadric containing $\mathcal{C}$ and not in a hyperbolic quadric spanning a space containing $\ell$, since the span of $\ell$ and $m$ meets $\mathcal{Q}$ in a cone. If $m$ is skew to $\pi$, then the span of $\ell$ and $m$ meets $\mathcal{Q}$ in a hyperbolic quadric.

For the following examples we require the concept of a flock. The study of flocks in their more general setting of circle planes is a thriving field of research, with many varied geometric structures closely associated to them, however here we need only the concept of a flock of the hyperbolic quadric $\mathcal{Q}^{+}$in $\mathrm{PG}(3, q)$, namely, a partition of the points of $\mathcal{Q}^{+}$into pairwise disjoint conics. We refer to the planes of $\mathrm{PG}(3, q)$ containing these conics as the planes of the flock. In fact, flocks of the hyperbolic quadric have been classified, being just the linear flocks, the Thas flocks (for odd $q$ ) and the exceptional flocks (for $q \in\{11,23,59\}$ ) (see [9] for a nice, short proof).

ExAmPLE 2. Let $\mathcal{F}$ be a flock of the hyperbolic quadric $\mathcal{Q}^{+}$, embedded as a hyperplane section of the parabolic quadric $\mathcal{Q}$ in $\mathrm{PG}(4, q)$ such that all the polar lines to the planes of $\mathcal{F}$ (with respect to $\mathcal{Q}$ ) are external (so that $q$ is congruent to 3 modulo 4 and $\mathcal{F}$ is not linear 
$[\mathbf{1}])$. Let $\Sigma$ be the space spanned by $\mathcal{Q}^{+}$. Then the set of hyperbolic quadrics spanning a space that meets $\Sigma$ in a plane of $\mathcal{F}$, together with $\mathcal{Q}^{+}$, gives rise to a set of reguli $\Pi$ satisfying the hypotheses of the theorem. To see this, let $m$ be a line of $\mathcal{Q}$ not in $\Sigma$. Then $m$ meets $\Sigma$ in a point $P$ of $\mathcal{Q}^{+}$, which lies in a unique plane $\pi$ of $\mathcal{F}$. The space spanned by $\pi$ and $m$ meets $\mathcal{Q}$ in a hyperbolic quadric, since if it met in a cone, the vertex would be polar to $\pi$, contrary to our hypothesis.

EXAMPLE 3. Let $\mathcal{F}$ be a flock of the hyperbolic quadric $\mathcal{Q}^{+}$, embedded as a hyperplane section of the parabolic quadric $\mathcal{Q}$ in $\mathrm{PG}(4, q)$ such that half of the polar lines to the planes of $\mathcal{F}$ (with respect to $\mathcal{Q}$ ) are external, and half are secant (so that either $\mathcal{F}$ is linear or $q$ is congruent to 1 modulo 4 and $\mathcal{F}$ is a Thas flock [1]). In particular, there is a common line $\ell$, external to $\mathcal{Q}$, on the planes of $\mathcal{F}$ with polar line secant. Let $\pi_{\ell}$ be the polar plane to $\ell$ (with respect to $\mathcal{Q}$ ), let $\mathcal{C}=\pi_{\ell} \cap \mathcal{Q}$ and let $\Sigma$ be the space spanned by $\mathcal{Q}^{+}$. Then the set of hyperbolic quadrics spanning a space that meets $\Sigma$ in a plane of $\mathcal{F}$, together with $\mathcal{Q}^{+}$and the hyperbolic quadrics containing $\mathcal{C}$, gives rise to a set of reguli $\Pi$ satisfying the hypotheses of the theorem. To see this, let $m$ be a line not in $\Sigma$. Then $m$ meets $\Sigma$ in a point $P$ of $\mathcal{Q}^{+}$, which lies in a unique plane $\pi$ of $\mathcal{F}$. Now either the space spanned by $\pi$ and $m$ meets $\mathcal{Q}$ in a hyperbolic quadric, or it meets it in a cone. In the latter case, the vertex of the cone would be polar to $\ell$ and so on $\mathcal{C}$, in which case $m$ and $\pi$ span a space meeting $\mathcal{Q}$ in a hyperbolic quadric containing $\mathcal{C}$.

When the third construction is applied to a linear flock, the first construction arises. We should also mention a connection here with a "symplectic polarity paired spread" in the work of Cossidente, Culbert, Ebert, and Marino [6]. A symplectic polarity paired spread corresponds to an ovoid of the Klein quadric under the Klein correspondence on which a reflection acts fixed-point-freely. The polars of the lines spanned by the orbits are then a set of $\left(q^{2}+1\right) / 2$ hyperbolic quadric 3-spaces of the $Q(4, q)$ on the axis of the reflection, pairwise meeting in conic planes (and conversely). The reguli of these hyperbolic quadrics are then a partition of the lines of $Q(4, q)$ into regulus-opposite regulus pairs (and conversely).

\section{7. $m$-ovoids of $H\left(3, q^{2}\right)$ and $H\left(4, q^{2}\right)$}

In $H\left(3, q^{2}\right)$, it is always possible to find a partition of the set of points into ovoids. Such a partition is called a fan and a construction can be found in $[\mathbf{3}]$. Hence, we have that $m$-ovoids of $H\left(3, q^{2}\right)$ exist for all $m$ with $0 \leq m \leq q^{2}+1$. It is well-known that there are no ovoids of $H\left(4, q^{2}\right)$ (see $[\mathbf{1 9}$, Section 4$]$ ). We now prove give a sufficient condition for an $m$-ovoid of $H\left(4, q^{2}\right)$ to exist.

TheOREM 7.1. Any $m$-ovoid of $H\left(4, q^{2}\right)$ has $m>\sqrt{q}$.

Proof. By Corollary 4.7, an $m$-ovoid of $H\left(4, q^{2}\right)$ gives a set of $u=\left(q^{5}+1\right) m$ points of $\mathrm{PG}\left(4, q^{2}\right)$ of type $\left(h_{1}, h_{2}\right)$ with respect to hyperplanes where $h_{1}=m\left(q^{3}+1\right)-q^{3}$ and $h_{2}=m\left(q^{3}+1\right)$. This gives a strongly regular graph (see [5]) on $N=q^{10}$ vertices with valency $K=u\left(q^{2}-1\right)$ with the number $\lambda$ of vertices adjacent to a pair of adjacent vertices being $K^{2}+3 K-q^{2}\left(w_{1}+w_{2}\right)-K q^{2}\left(w_{1}+w_{2}\right)+q^{4} w_{1} w_{2}$, where $w_{1}=u-h_{1}$ and $w_{2}=u-h_{2}$. So $\lambda=m^{2}\left(q^{2}-1\right)^{2}+3 m\left(q^{2}-1\right)-q^{5}$. The condition $\lambda \geq 0$ gives $m>\sqrt{q}$.

\section{Some more examples of intriguing sets}

Here we give some examples of intriguing sets that (at the moment) do not belong to an infinite family of examples. We use superscripts to denote multiplicity in our multisets.

EXAMPLE 4 (Tight sets of $W(3,7)$ ). Via its fully deleted permutation module, $A_{7}$ acts as a subgroup of $\mathrm{PGO}(5,7)$ and so as a subgroup of $\operatorname{PSp}(4,7)$. It has two orbits on points (see $[\mathbf{1 2}$, 
Remarks 1.3]) of lengths $\{120,280\}$, and hence each of these is intriguing. Thus the first orbit is 15-tight. The subgroup $A_{6}$ of $A_{7}$ has four orbits on points of $W(3,7)$ of lengths $\left\{40,120^{3}\right\}$, with the orbit of length 40 being 5-tight, and one orbit of length 120 being 15-tight (and having stabiliser $A_{7}$ ). The union of the remaining 2 orbits is the set of points covered by a partial spread of 30 lines of $W(3,7)$.

EXAMPLE 5 ( $m$-ovoids of $Q(4, q))$. A number of examples of $m$-ovoids of $Q(4, q)$ have been found with the use of a computer, for some small values of $q$.

For $q=5$, let $\mathcal{Q}^{+}$be a 3-dimensional hyperbolic quadric embedded in $Q(4,5)$ as a hyperplane section. Then the stabiliser in $\operatorname{PGO}(5,5)$ of $\mathcal{Q}^{+}$has two conjugacy classes of subgroups isomorphic to $\operatorname{GL}(2,3)$, exactly one of which has orbits on the points of $Q(4,5)$ of lengths $\left\{8,12^{3}, 16,24^{2}, 48\right\}$. The union of a particular choice of these orbits of lengths $12,16,24$ is a 2-ovoid of $Q(4,5)$.

For $q=7$, let $\mathcal{Q}^{-}$be a 3-dimensional elliptic quadric embedded in $Q(4,7)$ as a hyperplane section. Then the stabiliser in $\operatorname{PGO}(5,7)$ of $\mathcal{Q}^{-}$has two conjugacy classes of subgroups of order 96 with orbits on the points of $Q(4,7)$ of lengths $\left\{2,6,8,24^{4}, 48^{6}\right\}$. These subgroups are isomorphic to $C_{24} \rtimes\left(C_{2} \times C_{2}\right)$, and exactly one of them has orbits on lines of $Q(4,7)$ of lengths $\left\{16,48^{2}, 96^{3}\right\}$. The union of a particular choice of orbits on points of this subgroup of lengths $6,24,24,48,48$ is a 3 -ovoid of $Q(4,7)$.

For $q=9$, let $\mathcal{Q}^{-}$be a 3 -dimensional elliptic quadric embedded in $Q(4,9)$ as a hyperplane section. Then the stabiliser in $\operatorname{P\Gamma O}(5,9)$ of $\mathcal{Q}^{-}$has two conjugacy classes of dihedral subgroups of order 82 , exactly one of which has orbits on the points of $Q(4,9)$ of lengths $\left\{41^{10}, 82^{5}\right\}$. The union of a particular choice of these orbits of lengths $41,41,41,41,82$ is a 3 -ovoid of $Q(4,9)$. In fact, $\mathcal{Q}^{-}$is the union of two further orbits of lengths 41,41 , and is hence disjoint from the 3 -ovoid, giving rise to a partition of $Q(4,9)$ into a 1-ovoid, 3-ovoid and 6-ovoid.

For $q=11$, let $\mathcal{Q}^{-}$be a 3 -dimensional elliptic quadric embedded in $Q(4,11)$ as a hyperplane section. Then the normaliser of a Sylow 5-subgroup of the stabiliser in $\operatorname{PGO}(5,11)$ of $\mathcal{Q}^{-}$has two conjugacy classes of subgroups of order 240 with orbits on the points of $Q(4,11)$ of lengths $\left\{2,10,12,60^{6}, 120^{9}\right\}$. These subgroups are isomorphic to $C_{60} \rtimes\left(C_{2} \times C_{2}\right)$, and exactly one of them has orbits on lines of $Q(4,11)$ of lengths $\left\{24,120^{2}, 240^{5}\right\}$. The union of a particular choice of orbits on points of this subgroup of lengths 10,60,60,120,120,120,120 is a 5-ovoid of $Q(4,11)$.

Note that for $Q(4, q)$, we have the existence for all $q$ of 1-ovoids and their complement $q$-ovoids, and as mentioned in Section 3, we have the existence for all odd $q$ of $(q+1) / 2$-ovoids. Along with the few examples for small $q$ given above, and their complements, we summarise in the following table, for small $q$, the values of $m$ for which $m$-ovoids of $Q(4, q)$ are known to exist.

\begin{tabular}{|c|c|c|}
\hline$q$ & Known $m$ & Unknown $m$ \\
\hline 3 & $1,2,3$ & - \\
5 & $1,2,3,4,5$ & - \\
7 & $1,3,4,5,7$ & 2,6 \\
9 & $1,3,4,5,6,7,9$ & 2,8 \\
11 & $1,5,6,7,11$ & $2,3,4,8,9,10$ \\
\hline
\end{tabular}

TABLE $1 . m$-ovoids of $Q(4, q)$

\section{Open problems and conjectures}

In this paper, we have constructed $m$-ovoids for $W(3, q), q$ odd for all possible even integers $m$, but for odd $m$, the situation is unclear. The dual of a hemisystem of lines of $Q(4,5)$ is a 
3-ovoid of $W(3,5)$, which we constructed in Section 6. We conjecture that 3-ovoids of $W(3, q)$ do not exist in larger characteristic.

QUESTION 9.1. Is there a 3-ovoid of $W(3, q)$ for $q$ an odd prime power greater than 5 ?

Recall from Theorem 7.1, that an $m$-ovoid of $H\left(4, q^{2}\right)$ must have $m>\sqrt{q}$. However, it is not known whether $m$-ovoids or $m$-covers exist in $H\left(4, q^{2}\right)$.

Question 9.2.

(a) Do there exist $m$-ovoids of $H\left(4, q^{2}\right)$ for $\sqrt{q}<m<q^{2}+1$ ?

(b) Do there exist $m$-covers of $H\left(4, q^{2}\right)$ for $0<m<q+1$ ?

We asked above whether 3-ovoids of $W(3, q)$ exist, and we established in Section 5 that $m$-ovoids of $W(3, q), q$ odd, exist for all even $m$. Similarly, in Section 8 , we saw that $m$-ovoids of $Q(4, q)$ exist for several small values of $m$. The problem below asks whether we can complete the spectral problem of the existence of $m$-ovoids in these generalised quadrangles.

\section{QUESTION 9.3.}

(a) For what values of $m$ do $m$-ovoids of $W(3, q)$ exist?

(b) For what values of $m$ do m-ovoids of $Q(4, q)$ exist?

\section{References}

[1] Laura Bader, Nicola Durante, Maska Law, Guglielmo Lunardon, and Tim Penttila. Flocks and partial flocks of hyperbolic quadrics via root systems. J. Algebraic Combin., 16(1):21-30, 2002.

[2] Adriano Barlotti. Un'estensione del teorema di Segre-Kustaanheimo. Boll. Un. Mat. Ital. (3), 10:498-506, 1955.

[3] A. E. Brouwer and H. A. Wilbrink. Ovoids and fans in the generalized quadrangle Q(4,2). Geom. Dedicata, 36(1):121-124, 1990.

[4] A. A. Bruen and Keldon Drudge. The construction of Cameron-Liebler line classes in PG(3,q). Finite Fields Appl., 5(1):35-45, 1999.

[5] R. Calderbank and W. M. Kantor. The geometry of two-weight codes. Bull. London Math. Soc., 18(2):97$122,1986$.

[6] Antonio Cossidente, C. Culbert, Gary L. Ebert, and G. Marino. On m-ovoids of W $(3, q)$. submitted to Combinatorica.

[7] Antonio Cossidente and Tim Penttila. Hemisystems of the hermitian surface. submitted to J. London Math. Soc.

[8] Keldon Drudge. Proper 2-covers of PG(3,q), q even. Geom. Dedicata, 80(1-3):59-64, 2000.

[9] Nicola Durante and Alessandro Siciliano. (B)-geometries and flocks of hyperbolic quadrics. J. Combin. Theory Ser. A, 102(2):425-431, 2003.

[10] Gary L. Ebert. The completion problem for partial packings. Geom. Dedicata, 18(3):261-267, 1985.

[11] Gary L. Ebert. Partitioning projective geometries into caps. Canad. J. Math., 37(6):1163-1175, 1985.

[12] Martin W. Liebeck. The affine permutation groups of rank three. Proc. London Math. Soc. (3), 54(3):477$516,1987$.

[13] Gianfranco Panella. Caratterizzazione delle quadriche di uno spazio (tridimensionale) lineare sopra un corpo finito. Boll. Un. Mat. Ital. (3), 10:507-513, 1955.

[14] S. E. Payne and J. A. Thas. Finite generalized quadrangles, volume 110 of Research Notes in Mathematics. Pitman (Advanced Publishing Program), Boston, MA, 1984.

[15] Stanley E. Payne. Tight pointsets in finite generalized quadrangles. Congr. Numer., 60:243-260, 1987. Eighteenth Southeastern International Conference on Combinatorics, Graph Theory, and Computing (Boca Raton, Fla., 1987).

[16] Beniamino Segre. Forme e geometrie hermitiane, con particolare riguardo al caso finito. Ann. Mat. Pura Appl. (4), 70:1-201, 1965.

[17] E. E. Shult and J. A. Thas. $m$-systems of polar spaces. J. Combin. Theory Ser. A, 68(1):184-204, 1994.

[18] J. A. Thas. Ovoidal translation planes. Arch. Math. (Basel), 23:110-112, 1972.

[19] J. A. Thas. Ovoids and spreads of finite classical polar spaces. Geom. Dedicata, 10(1-4):135-143, 1981.

[20] J. A. Thas. Interesting pointsets in generalized quadrangles and partial geometries. Linear Algebra Appl., 114/115:103-131, 1989. 
School of Mathematics and Statistics, University of Western Australia, 35 Stirling HighWay, Crawley, Western Australia 6009

E-mail address: john.bam@maths.uwa.edu.au, maska@maths.uwa.edu.au, penttila@maths.uwa.edu.au 